\title{
Application of the Proximal Isovelocity Surface Area Method for Estimation of the Effective Orifice Area in Aortic Stenosis
}

\section{Masahiro Nakabachi}

Hokkaido University Hospital: Hokkaido Daigaku Byoin

Hiroyuki Iwano ( $\nabla$ h-iwano@med.hokudai.ac.jp )

Hokkaido University https://orcid.org/0000-0002-1267-8260

\section{Michito Murayama}

Hokkaido University Hospital: Hokkaido Daigaku Byoin

\section{Hisao Nishino}

Hokkaido University Hospital: Hokkaido Daigaku Byoin

\section{Shinobu Yokoyama}

Hokkaido University Hospital: Hokkaido Daigaku Byoin

\section{Shingo Tsujinaga}

Hokkaido University: Hokkaido Daigaku

\section{Yasuyuki Chiba}

Hokkaido University: Hokkaido Daigaku

\section{Suguru Ishizaka}

Hokkaido University: Hokkaido Daigaku

Ko Motoi

Hokkaido University: Hokkaido Daigaku

\section{Kazunori Okada}

Hokkaido University: Hokkaido Daigaku

\section{Sanae Kaga}

Hokkaido University: Hokkaido Daigaku

\section{Mutsumi Nishida}

Hokkaido University Hospital: Hokkaido Daigaku Byoin

\section{Takanori Teshima}

Hokkaido University: Hokkaido Daigaku

\section{Toshihisa Anzai}

Hokkaido University: Hokkaido Daigaku 
Keywords: Doppler echocardiography, aortic stenosis, proximal isovelocity surface area method, valve orifice area

Posted Date: March 6th, 2021

DOI: https://doi.org/10.21203/rs.3.rs-287004/v1

License: (1) This work is licensed under a Creative Commons Attribution 4.0 International License. Read Full License

Version of Record: A version of this preprint was published at Heart and Vessels on September 25th, 2021. See the published version at https://doi.org/10.1007/s00380-021-01945-5. 


\section{Abstract}

Purpose: Because existence of high flow velocity at the left ventricular outflow tract (LVOT) potentially causes an overestimation of effective orifice area (EOA) by continuity equation in aortic stenosis (AS), we tested the proximal isovelocity surface area (PISA) method as an alternative tool for AS.

Methods: EOA was calculated using the continuity equation $\left(\mathrm{EOA}_{\text {Cont }}\right)$ and PISA method (EOA PISA), respectively, in 114 patients with at least moderate AS. The geometric orifice area (GOA) was also measured in 51 patients who also underwent three-dimensional transesophageal echocardiography (TEE). Patients were divided into two groups according to the median LVOT flow velocity.

Results: Feasibility of EOA $A_{\text {PISA }}$ was $95 \%$ in the 114 patients. While there was a strong correlation between

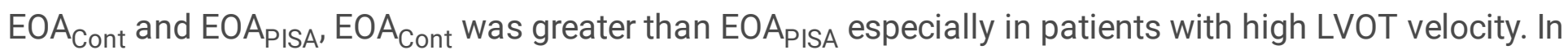
TOE cohort, both EOA Cont $_{\text {and EOA }}$ ISA similarly correlated with GOA. However, a fixed bias, which is supposed to exist in AS, was observed only between EOA $A_{P I S A}$ and GOA with smaller EOA $A_{\text {PISA }}$ than GOA.

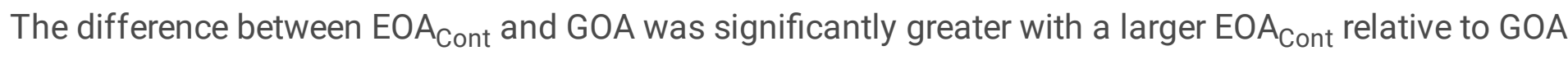
in patients with high LVOT velocity than in those without $\left(0.16 \pm 0.25 \mathrm{vs}-0.07 \pm 0.10 \mathrm{~cm}^{2}, P<0.001\right)$. In contrast, the difference between EOAPISA and GOA was consistent in both groups $(-0.07 \pm 0.12$ vs $\left.-0.07 \pm 0.15 \mathrm{~cm}^{2}, P=0.936\right)$.

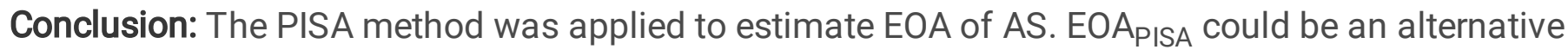
parameter for AS severity grading in patients with high LVOT velocity in whom EOA Cont $_{\text {would }}$ overestimate the orifice area.

\section{Introduction}

Aortic stenosis (AS) is the most common valvular heart disease in developed countries and its prevalence is growing with the aging population. [1,2] In clinical practice, accurate assessment of AS severity is essential for appropriate management and therapeutic decision making. Echocardiography is a key tool in determining AS severity, and the effective orifice area (EOA) assessed by the continuity equation is one of the main variables that is recommended for assessment in the guidelines. [3] Owing to its feasibility and reproducibility, the continuity equation is widely used in the assessment of AS severity; however, some potential sources of error have been identified. Among them, increased flow velocity at the left ventricular outflow tract (LVOT) due to upper septal hypertrophy (USH) was reported to cause overestimation of the stroke volume (SV) and subsequent EOA. [4,5] Because USH is common in elderly AS patients, [6] an alternative method to quantify the EOA is expected to improve the diagnostic accuracy in these patients.

The proximal isovelocity surface area (PISA) method is widely used to quantify the effective regurgitant orifice area in valve regurgitation. [7] By applying the PISA method to AS, the EOA could be estimated in patients who are potentially unsuitable for applying continuity equation. In terms of the 
application of the PISA method for the stenotic valve, it has been used for mitral stenosis; [8] however, the feasibility and diagnostic accuracy of the PISA method for AS severity has not been reported.

Accordingly, the present study aimed to validate the PISA method for the estimation of EOA in AS and to test whether the PISA method could overcome the overestimation of the continuity equation caused by high flow velocity at the LVOT in patients with AS.

\section{Methods}

\section{Study Population}

We prospectively enrolled 160 consecutive patients who had been diagnosed with at least moderate AS (peak velocity across the aortic valve $>3.0 \mathrm{~m} / \mathrm{s}$ or EOA $<1.5 \mathrm{~cm}^{2}$ ) referred for transthoracic echocardiography from May 2018 to March 2019 at Hokkaido University Hospital. After the exclusion of patients with irregular heart rhythm due to arrhythmias $(\mathrm{N}=38)$ and those with poor echocardiographic images $(\mathrm{N}=8)$ in whom EOA by continuous equation could not be obtained, 114 patients were included in the final analysis. In 51 of the 114 patients who also underwent transesophageal echocardiography (TEE) after transthoracic echocardiographic examination (median distance: 27 days: TEE cohort), the geometric orifice area (GOA) of the aortic valve was used as the reference for the valve area. Study approval was obtained from the institutional review board of Hokkaido University Hospital (No. 0180179) and an opportunity to opt-out was given to each participant through a published disclosure document on the web site of the institute.

\section{Two-Dimensional and Doppler Echocardiography}

Comprehensive transthoracic echocardiography was performed in the left decubitus position using a commercially available ultrasound equipment system (Vivid E9, GE Healthcare, Horton, Norway; iE33, Philips Medical Systems, Andover, Massachusetts; Acuson SC2000 prime, Siemens Healthineers, Erlangen, Germany; Aplio Artida, Canon Medical Systems, Otawara, Japan) according to the guidelines. [9] Left ventricular (LV) mass was determined using the Devereux formula. LV ejection fraction (EF) was calculated by using the biplane method of disks. USH was defined as a ratio of upper septal wall thickness to mid-septal wall thickness ${ }^{31}$ 1.3. The maximal aortic valve velocity (Vmax) was measured using continuous-wave Doppler imaging in the view that showed the highest flow velocity, and the mean transvalvular gradient was estimated based on the simplified Bernoulli equation. The LVOT diameter was measured in the parasternal long-axis view. Flow velocity at the LVOT was measured using pulsed-wave Doppler imaging in the apical long-axis view, in which the sample volume was placed just proximal to the region of flow acceleration, and the time velocity integral (TVI $\mathrm{LVOT}$ ) was measured. SV was then calculated as $\mathrm{SV}_{\text {Cont }}=(\mathrm{LVOT} \text { diameter } / 2)^{2}{ }^{\prime} \mathrm{P}^{\prime} \mathrm{TVI}_{\mathrm{LVOT}}$. EOA of the aortic valve was calculated using the continuity equation: $\mathrm{EOA}_{\text {Cont }}=\mathrm{SV} / \mathrm{TVI}_{\mathrm{AV}}$, where $\mathrm{TVI}_{\mathrm{AV}}$ was the time velocity integral of the maximal

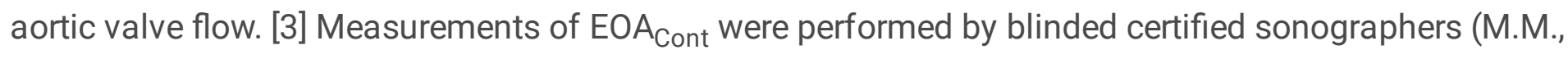
S.Y., H.N., M.N., and S.K.) and verified by a board certified reader (H.I.). 


\section{PISA Method for Estimation of EOA}

Measurements of EOA by PISA methods were performed by the sonographers (M.M. or M.N) who were blinded to the results of clinical and echocardiographic parameters. To obtain a clear visualization of the proximal convergence of the aortic stenotic jet, a cut plane was selected in each patient from the apical long-axis, apical four-chamber, right parasternal long-axis, and suprasternal long-axis views (Figure 1A). Care was taken to minimize the angle between the centerline of the flow convergence and ultrasound beam. Using a magnified image, the appearance of PISA was optimized by shifting the baseline of the color Doppler scale to forward direction to adjust for aliasing velocity around 50 to $70 \mathrm{~cm} / \mathrm{s}$ as appropriate. The radius of PISA ( $r$ ) was then measured, and the instantaneous flow rate was calculated as $2 \Pi r^{2}$ ' aliasing velocity $(\mathrm{mL} / \mathrm{s})$. EOA was then calculated using the PISA method: EOA $_{\text {PISA }}=$ flow rate/Vmax $\left(\mathrm{cm}^{2}\right)$ based on the principle of conservation of mass (Figure 1). The SV was also calculated by EOA PISA $^{\prime} \mathrm{TVI}_{\mathrm{AV}}\left(\mathrm{SV} \mathrm{PISA}_{\mathrm{P}}\right)$.

\section{Measurement of GOA}

Transesophageal echocardiographic images were obtained using an iE33 (Philips Medical Systems, Andover, Massachusetts) with X7-2t probe or an Acuson SC2000 prime (Siemens Healthineers, Erlangen, Germany) with Z6Ms probe. At first, the two-dimensional images were clearly obtained in the midesophageal long-axis and short-axis views. Then volume data sets were obtained using four-beat fullvolume mode $(\mathrm{N}=38$, median volume rate: $27 \mathrm{~Hz})$ or the live three-dimensional zoom mode $(\mathrm{N}=13$, median volume rate: $21 \mathrm{~Hz}$ ) focused on the aortic valve complex. During acquisition of full volume images, gain and compression settings were optimized to display a magnified image of the aortic valve. After choosing the mid-systolic frame, in which maximal aortic valve excursion was observed, fine adjustments of the short-axis plane were performed to obtain the smallest aortic valve orifice and the GOA was measured (Figure 1B). All the measurements of GOA were performed by trained cardiovascular physicians (S.I, Y.C., and S.T.) and designated by a certified reader (H.I.).

\section{Classifications of the Patients}

To test the influence of LVOT flow velocity and LV morphology on the accuracy of measuring the EOA, we divided the patients by the median value of LVOT flow velocity and the presence or absence of USH and compared the differences in $\mathrm{EOA}_{\mathrm{Cont}}$ to aortic valve area-related variables between the groups.

\section{Statistical Analysis}

Continuous variables are expressed as mean \pm standard deviation and compared by paired or unpaired $\mathrm{t}$ test. Categorical data are presented as number (percentage). Agreements between aortic valve areas assessed using different methods were evaluated using Pearson's correlation coefficient and BlandAltman plot analysis. The Bland-Altman procedure for comparing two methods was also used to determine the bias ( $95 \%$ confidential interval: $\mathrm{Cl}$ ). A two-sided $P$ value $<0.05$ was considered statistically 
significant. All statistical analyses were performed using JMP version 14.0 (SAS Institute Inc., Cary, North Carolina).

\section{Results}

\section{Patient Characteristics}

The clinical and echocardiographic characteristics of the studied patients are summarized in Table 1. In the overall cohort, relatively young patients with AS who were predominantly female were enrolled. More than half of the patients had experienced some symptoms of heart failure. While $69 \%$ of the patients were diagnosed as hypertension, the blood pressure was well controlled at $<140 \mathrm{mmHg}$ during the echocardiographic examination[10] in the majority of these patients. LV hypertrophy defined as an increased LV mass index was observed in $57 \%$ and USH was found in $46 \%$ of patients. Since the most common reason for TEE was to decide on surgical or transcatheter aortic valve replacement, the 51 patients in the TEE cohort consisted of elderly patients with a high frequency of heart failure symptoms and more advanced AS severity, whereas the LV geometry was similar to that in the overall cohort.

\section{Comparisons of EOA cont $_{\text {and EOA }}$ ISA}

According to $\mathrm{EOA}_{\text {Cont }}, 79$ patients were classified as severe AS and remaining 35 patients as moderate AS. In the 114 patients in whom the severity of AS was assessed based on EOA Cont, $_{\text {, estimation of }}$ EOA $_{\text {PISA }}$ was possible in 108 patients (95\%) and impossible due to inadequate image quality of PISA radius in 6 patients (5\%). Although there was a strong correlation between EOA $\mathrm{A}_{\text {Cont }}$ and EOA $\mathrm{AISA}_{\text {(Figure }}$ 2A), EOA $A_{P I S A}$ was statistically significantly smaller than EOA cont (Figure 2B). Accordingly, 14 of 33 patients (42\%) who were diagnosed with moderate AS based on $\mathrm{EOA}_{\text {Cont }}$ was classified with severe based on EOA $A_{P I S A}$. Interestingly, the difference between $E O A_{\text {Cont }}$ and EOA $A_{P I S A}$ was greater in patients with USH (Figure 2C). Further, the difference was also greater in patients with high LVOT flow velocity than in those without (Figure 2D).

\section{TEE Cohort}

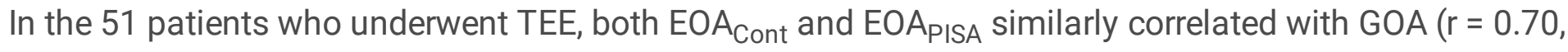
$P<0.001$ and $r=0.77, P<0.001$, respectively) (Figure 3A, C). However, a negative fixed bias, which is hydrodynamically supposed to exist between effective and geometric orifice areas, was not observed between $\mathrm{EOA}_{\mathrm{Cont}}$ and GOA ( $95 \% \mathrm{Cl}-0.03$ to 0.08 ; Figure 3B). In contrast, there was a negative fixed bias between EOAPISA and GOA, with smaller EOA $A_{P I S A}$ than GOA (95\% Cl-0.11 to -0.03; Figure 3D). Importantly, the difference between $\mathrm{EOA}_{\mathrm{Cont}}$ and GOA was significantly greater with a larger $\mathrm{EOA}_{\mathrm{Cont}}$ than GOA in patients with high LVOT flow velocity than in those without (Figure 4A). In contrast, the difference between EOAPISA and GOA was consistent regardless of the LVOT flow velocity (Figure 4B). Similar results were observed in the comparison between patients with and without USH (Figure 4C, D). In a typical case with severe AS (GOA: $0.70 \mathrm{~cm}^{2}$ ) complicated by mid-ventricular obstruction, a clear 
difference was observed between EOA Cont and EOAPISA $\left(1.46 \mathrm{~cm}^{2}\right.$ vs $\left.0.55 \mathrm{~cm}^{2}\right)$, suggesting an

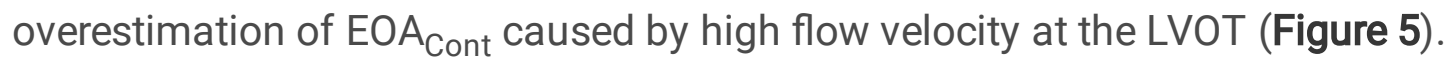

\section{Reproducibility Analysis}

The reproducibility for EOAPISA was assessed in 30 randomly selected patients. Two independent observers (M.N. and M.M.) analyzed the same cine loops of zoomed color Doppler images and one blinded observer (M.N.) repeated the analysis on a separate day. The intra- and interobserver intraclass correlation coefficients of EOA $\mathrm{PISA}_{\text {A }}$ were 0.98 and 0.95 , respectively.

\section{Discussion}

In the present study, we tested the feasibility and reliability of EOA $A_{P I S A}$ in AS patients and found that 1) estimation of EOA using the PISA method was possible in up to $95 \%$ of AS patients, in whom EOA could be obtained, with acceptable consistency with EOA Cont, $_{2}$ 2) EOA

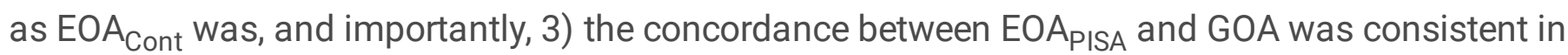
patients with high LVOT flow velocity and those with USH in whom EOA ${ }_{\text {Cont }}$ potentially overestimated the valve orifice area. Although the PISA method has been applied for other valvular heart diseases, [11-14] this is the first study to demonstrate the feasibility and reliability of this method in AS.

\section{PISA Method for Assessment of AS Severity}

In the same manner as the continuity equation, the PISA method is based on the principle of conservation of mass, where flow rate during the ejection period through the proximal isovelocity surface is equal to that through effective orifice area of the narrowing orifice. The physical basis of this method is quite solid, and use of the PISA method to quantify the severity of mitral regurgitation, mitral stenosis, and aortic regurgitation is recommended by the guidelines. $[15,16]$ However, the use of PISA method for AS

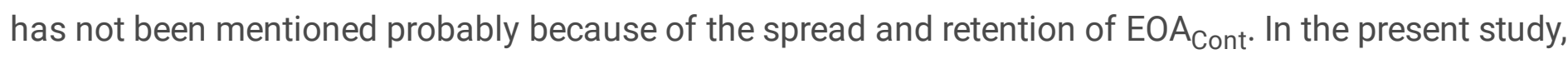
we demonstrated an acceptable feasibility and reproducibility of EOA $A_{\text {ISA }}$ in clinical AS patients.

\section{EOA $_{\text {Cont }}$ for Assessment of AS Severity}

Transvalvular gradients and $\mathrm{EOA}_{\mathrm{Cont}}$ are the principal measures for the echocardiographic grading of severity in AS. [3] Among them, estimation of EOA is important because transvalvular gradients vary depending on the flow conditions. [17] An accurate estimation of EOA $\mathrm{Cont}_{\text {is }}$ thus mandatory for managing patients with AS; however, sources of errors have been noted in the measurement of its components. $[18,19]$ As the LVOT cross-sectional area is calculated based on the anterior to posterior diameter assuming a circular shape, it can underestimate the area, which has an elliptical shape in most patients. [20-24] At the same time, a study showed that $\mathrm{TVI}_{\mathrm{LVOT}}$ was overestimated using pulsed-wave Doppler, resulting in compensation for the underestimation of the LVOT area with subsequent good concordance between EOA $\mathrm{Cont}_{\text {t }}$ and cardiac magnetic resonance-derived EOA. [5] This overestimation of 
$\mathrm{TVI}_{\mathrm{LVOT}}$ was thought to be due to the alteration of the flow velocity profile from flat to skewed in the presence of high flow velocity at the LVOT, as seen in USH. [5,25] Koto et al. [4] demonstrated that AS patients with USH had greater SV and EOA calculated using the Doppler method than those without. Consistent with these previous findings, when SV was compared between patients with and without USH or those with and without high LVOT flow velocity, apparent differences were observed only in SV Cont $_{\text {but }}$ not in SV $_{\text {PISA }}$ between groups (Table 3). Therefore, the significant differences in EOA Cont and EOA (Figure $2 \mathrm{C}$ and $\mathrm{D}$ ) and those in $\mathrm{EOA}_{\mathrm{Cont}}$ and GOA (Figure $4 \mathrm{~A}$ and $\mathrm{C}$ ) between patients with and without USH or those with and without high LVOT flow velocity, along with the absence of a negative fixed bias between $\mathrm{EOA}_{\text {Cont }}$ and GOA, suggests overestimation of EOA $\mathrm{Cont}_{\text {in }}$ in patients with high LVOT flow velocity.

\section{Clinical Implication}

Owing to the increasing elderly population with AS, a substantial population of the patients could show a narrowing LV cavity with USH, resulting in increased LVOT flow velocity. [26,27] As observed in a previous study[4] and ours, the continuity equation would overestimate SV and subsequent EOA in these patients. In typical cases with USH or LVOT obstruction, it is generally impossible to avoid sampling high flow speed by placing the sample volume at anywhere of the proximal site of the aortic valve annulus due to the turbulent flow. In that case, EOA $A_{\text {PISA }}$ could be an alternative parameter to determine AS severity. $E_{\text {EOA }}$ PISA is expected to be particularly effective in patients showing EOA $\mathrm{A}_{\text {Cont }}$ that is larger than expected from the transvalvular flow velocity as shown in Figure 5. As the diagnosis of severity is critical in AS,[3] many reliable and feasible variables are needed for its assessment. EOAPISA is in line with other recently proposed markers of AS severity. $[4,27,28]$

\section{Study Limitations}

This study had several limitations. First, the relatively small number of studied patients limits the strength of our findings; thus, the results of our study should be confirmed in a larger study. Second, test-retest reproducibility between the two examinations was not assessed, although the consistency of the EOAPISA obtained from the same cine loops was acceptable. Third, while the PISA method assumes the shape of the proximal isovelocity area to be hemispherical, we could not confirm its exact geometry in AS. As noted in a recent computational simulation, [15] an ellipsoidal PISA geometry results in substantial underestimation. In addition, based on the funnel shape geometry of the stenotic orifice, PISA might require correction based on an angle such as the PISA method in mitral stenosis. However, shape of stenotic orifice in AS, especially in sclerotic degenerative valves, is symmetrical and far from funnel shape compared to that of mitral stenosis, which might have reduced the alteration of PISA geometry in AS. In fact, the EOAPISA was $10-30 \%$ smaller than GOA in our study, which was within the previously reported difference ranges between EOA and GOA (10\% to 30\%),[29] and can justify the application of PISA method without angle correction for AS.

\section{Conclusions}


In conclusion, the PISA method was applied for the estimation of EOA in patients with AS. EOAPISA can be used as an alternative variable when assessing the AS severity in patients with high LVOT flow velocity or USH in whom EOA $\mathrm{Eont}_{\mathrm{t}}$ would potentially overestimate the orifice area.

\section{Declarations}

\section{Acknowledgements}

We would like to thank Honyaku Center Inc. (www.honyakuctren.com) for English language editing.

\section{Compliance of Ethical Standards}

As mentioned above, the study protocol has been approved by the ethics committee in our hospital and therefore was performed in accordance with the ethical standards laid down in the 1964 Declaration of Helsinki and its later amendments.

\section{References}

1. Carabello BA, Paulus WJ (2009) Aortic stenosis. The Lancet 373 (9667):956-966. doi:10.1016/s0140-6736(09)60211-7

2. Carabello BA (2002) Clinical practice. Aortic stenosis. N Engl J Med 346 (9):677-682. doi:10.1056/NEJMcp010846

3. Baumgartner $\mathrm{H}$, Hung J, Bermejo J, Chambers JB, Edvardsen T, Goldstein S, Lancellotti P, LeFevre M, Miller F, Jr., Otto CM (2017) Recommendations on the Echocardiographic Assessment of Aortic Valve Stenosis: A Focused Update from the European Association of Cardiovascular Imaging and the American Society of Echocardiography. J Am Soc Echocardiogr 30 (4):372-392. doi:10.1016/j.echo.2017.02.009

4. Koto D, Izumo M, Machida T, Suzuki K, Yoneyama K, Suzuki T, Kamijima R, Kobayashi Y, Harada T, Akashi YJ (2018) Geometry of the left ventricular outflow tract assessed by 3D TEE in patients with aortic stenosis: impact of upper septal hypertrophy on measurements of Doppler-derived left ventricular stroke volume. J Echocardiogr 16 (4):162-172. doi:10.1007/s12574-018-0383-7

5. Garcia J, Kadem L, Larose E, Clavel MA, Pibarot P (2011) Comparison between cardiovascular magnetic resonance and transthoracic Doppler echocardiography for the estimation of effective orifice area in aortic stenosis. J Cardiovasc Magn Reson 13:25. doi:10.1186/1532-429X-13-25

6. Diaz T, Pencina MJ, Benjamin EJ, Aragam J, Fuller DL, Pencina KM, Levy D, Vasan RS (2009) Prevalence, clinical correlates, and prognosis of discrete upper septal thickening on echocardiography: the Framingham Heart Study. Echocardiography 26 (3):247-253. doi:10.1111/j.1540-8175.2008.00806.x

7. Xie GY, Berk MR, Hixson CS, Smith AC, DeMaria AN, Smith MD (1995) Quantification of mitral regurgitant volume by the color Doppler proximal isovelocity surface area method: a clinical study. $J$ 
Am Soc Echocardiogr 8 (1):48-54. doi:10.1016/s0894-7317(05)80357-8

8. Rifkin RD, Harper K, Tighe D (1995) Comparison of proximal isovelocity surface area method with pressure half-time and planimetry in evaluation of mitral stenosis. J Am Coll Cardiol 26 (2):458-465. doi:10.1016/0735-1097(95)80023-a

9. Lang RM, Badano LP, Mor-Avi V, Afilalo J, Armstrong A, Ernande L, Flachskampf FA, Foster E, Goldstein SA, Kuznetsova T, Lancellotti P, Muraru D, Picard MH, Rietzschel ER, Rudski L, Spencer KT, Tsang W, Voigt JU (2015) Recommendations for cardiac chamber quantification by echocardiography in adults: an update from the American Society of Echocardiography and the European Association of Cardiovascular Imaging. J Am Soc Echocardiogr 28 (1):1-39 e14. doi:10.1016/j.echo.2014.10.003

10. Nishimura RA, Otto CM, Bonow RO, Carabello BA, Erwin JP, 3rd, Guyton RA, O'Gara PT, Ruiz CE, Skubas NJ, Sorajja P, Sundt TM, 3rd, Thomas JD (2014) 2014 AHA/ACC guideline for the management of patients with valvular heart disease: a report of the American College of Cardiology/American Heart Association Task Force on Practice Guidelines. J Am Coll Cardiol 63 (22):e57-185. doi:10.1016/j.jacc.2014.02.536

11. Bargiggia GS, Tronconi L, Sahn DJ, Recusani F, Raisaro A, De Servi S, Valdes-Cruz LM, Montemartini C (1991) A new method for quantitation of mitral regurgitation based on color flow Doppler imaging of flow convergence proximal to regurgitant orifice. Circulation 84 (4):1481-1489. doi:10.1161/01.cir.84.4.1481

12. Shiota T, Jones M, Agler DA, McDonald RW, Marcella CP, Qin JX, Zetts AD, Greenberg NL, Cardon LA, Sun JP, Sahn DJ, Thomas JD (1999) New echocardiographic windows for quantitative determination of aortic regurgitation volume using color Doppler flow convergence and vena contracta. Am J Cardiol 83 (7):1064-1068. doi:10.1016/s0002-9149(99)00016-8

13. Rivera JM, Vandervoort PM, Mele D, Siu S, Morris E, Weyman AE, Thomas JD (1994) Quantification of tricuspid regurgitation by means of the proximal flow convergence method: a clinical study. Am Heart J 127 (5):1354-1362. doi:10.1016/0002-8703(94)90056-6

14. Messika-Zeitoun D (2003) Sequential assessment of mitral valve area during diastole using colour M-mode flow convergence analysis: new insights into mitral stenosis physiology. European Heart Journal 24 (13):1244-1253. doi:10.1016/s0195-668x(03)00208-2

15. Zoghbi WA, Adams D, Bonow RO, Enriquez-Sarano M, Foster E, Grayburn PA, Hahn RT, Han Y, Hung J, Lang RM, Little SH, Shah DJ, Shernan S, Thavendiranathan P, Thomas JD, Weissman NJ (2017) Recommendations for Noninvasive Evaluation of Native Valvular Regurgitation: A Report from the American Society of Echocardiography Developed in Collaboration with the Society for Cardiovascular Magnetic Resonance. J Am Soc Echocardiogr 30 (4):303-371. doi:10.1016/j.echo.2017.01.007

16. Baumgartner $H$, Hung J, Bermejo J, Chambers JB, Evangelista A, Griffin BP, lung B, Otto CM, Pellikka PA, Quinones M, American Society of E, European Association of E (2009) Echocardiographic 
assessment of valve stenosis: EAE/ASE recommendations for clinical practice. J Am Soc Echocardiogr 22 (1):1-23; quiz 101-102. doi:10.1016/j.echo.2008.11.029

17. deFilippi CR, Willett DL, Brickner ME, Appleton CP, Yancy CW, Eichhorn EJ, Grayburn PA (1995) Usefulness of dobutamine echocardiography in distinguishing severe from nonsevere valvular aortic stenosis in patients with depressed left ventricular function and low transvalvular gradients. Am J Cardiol 75 (2):191-194. doi:10.1016/s0002-9149(00)80078-8

18. Haugen BO, Berg S, Brecke KM, Torp H, Slordahl SA, Skaerpe T, Samstad SO (2002) Blood flow velocity profiles in the aortic annulus: a 3-dimensional freehand color flow Doppler imaging study. $J$ Am Soc Echocardiogr 15 (4):328-333. doi:10.1067/mje.2002.117292

19. Jainandunsing JS, Mahmood F, Matyal R, Shakil O, Hess PE, Lee J, Panzica PJ, Khabbaz KR (2013) Impact of three-dimensional echocardiography on classification of the severity of aortic stenosis. Ann Thorac Surg 96 (4):1343-1348. doi:10.1016/j.athoracsur.2013.05.018

20. Caballero L, Saura D, Oliva-Sandoval MJ, Gonzalez-Carrillo J, Espinosa MD, Garcia-Navarro M, Valdes M, Lancellotti P, de la Morena G (2017) Three-Dimensional Morphology of the Left Ventricular Outflow Tract: Impact on Grading Aortic Stenosis Severity. J Am Soc Echocardiogr 30 (1):28-35. doi:10.1016/j.echo.2016.10.006

21. Gaspar T, Adawi S, Sachner R, Asmer I, Ganaeem M, Rubinshtein R, Shiran A (2012) Threedimensional imaging of the left ventricular outflow tract: impact on aortic valve area estimation by the continuity equation. J Am Soc Echocardiogr 25 (7):749-757. doi:10.1016/j.echo.2012.05.001

22. Saitoh T, Shiota M, Izumo M, Gurudevan SV, Tolstrup K, Siegel RJ, Shiota T (2012) Comparison of left ventricular outflow geometry and aortic valve area in patients with aortic stenosis by 2-dimensional versus 3-dimensional echocardiography. Am J Cardiol 109 (11):1626-1631. doi:10.1016/j.amjcard.2012.01.391

23. Kamperidis V, van Rosendael PJ, Katsanos S, van der Kley F, Regeer M, Al Amri I, Sianos G, Marsan NA, Delgado V, Bax JJ (2015) Low gradient severe aortic stenosis with preserved ejection fraction: reclassification of severity by fusion of Doppler and computed tomographic data. Eur Heart J 36 (31):2087-2096. doi:10.1093/eurheartj/ehv188

24. Clavel MA, Malouf J, Messika-Zeitoun D, Araoz PA, Michelena HI, Enriquez-Sarano M (2015) Aortic valve area calculation in aortic stenosis by CT and Doppler echocardiography. JACC Cardiovasc Imaging 8 (3):248-257. doi:10.1016/j.jcmg.2015.01.009

25. Zhou YQ, Abassi I, Faerestrand S (1996) Flow velocity distributions in the left ventricular outflow tract and in the aortic annulus in patients with localized basal septal hypertrophy. Eur Heart J 17 (9):14041412. doi:10.1093/oxfordjournals.eurheartj.a015075

26. Pibarot P, Clavel MA (2015) Left ventricular outflow tract geometry and dynamics in aortic stenosis: implications for the echocardiographic assessment of aortic valve area. J Am Soc Echocardiogr 28 (11):1267-1269. doi:10.1016/j.echo.2015.09.006

27. Nabeshima Y, Nagata Y, Negishi K, Seo Y, Ishizu T, Sato K, Aonuma K, Koto D, Izumo M, Akashi YJ, Yamashita E, Oshima S, Otsuji Y, Takeuchi M (2018) Direct Comparison of Severity Grading Assessed 
by Two-Dimensional, Three-Dimensional, and Doppler Echocardiography for Predicting Prognosis in Asymptomatic Aortic Stenosis. J Am Soc Echocardiogr 31 (10):1080-1090 e1083.

doi:10.1016/j.echo.2018.05.006

28. Sato K, Seo Y, Ishizu T, Nakajima H, Takeuchi M, Izumo M, Suzuki K, Akashi YJ, Otsuji Y, Aonuma K (2016) Reliability of Aortic Stenosis Severity Classified by 3-Dimensional Echocardiography in the Prediction of Cardiovascular Events. Am J Cardiol 118 (3):410-417.

doi:10.1016/j.amjcard.2016.05.016

29. Gilon D, Cape EG, Handschumacher MD, Song J-K, Solheim J, VanAuker M, King MEE, Levine RA (2002) Effect of three-dimensional valve shape on the hemodynamics of aortic stenosis. Journal of the American College of Cardiology 40 (8):1479-1486. doi:10.1016/s0735-1097(02)02269-6

\section{Tables}

Table 1. Clinical and echocardiographic characteristics 


\begin{tabular}{|c|c|c|}
\hline & All $(N=108)$ & TEE cohort $(\mathrm{N}=51)$ \\
\hline Age, years & $78 \pm 12$ & $84 \pm 7$ \\
\hline Male, N (\%) & $41(38 \%)$ & $20(39 \%)$ \\
\hline Systolic blood pressure, $\mathrm{mmHg}$ & $131 \pm 19$ & $130 \pm 16$ \\
\hline Diastolic blood pressure, $\mathrm{mmHg}$ & $67 \pm 11$ & $66 \pm 10$ \\
\hline \multicolumn{3}{|l|}{ NYHA, N (\%) } \\
\hline 1 & $50(46 \%)$ & $7(14 \%)$ \\
\hline II & $52(48 \%)$ & $38(75 \%)$ \\
\hline III & $5(5 \%)$ & $5(10 \%)$ \\
\hline \multicolumn{3}{|l|}{ Comorbidity, N (\%) } \\
\hline Hypertension & $74(69 \%)$ & $41(80 \%)$ \\
\hline Diabetes mellitus & $28(26 \%)$ & $16(31 \%)$ \\
\hline Dyslipidemia & $53(49 \%)$ & $25(49 \%)$ \\
\hline \multicolumn{3}{|l|}{ Etiology, N (\%) } \\
\hline Tricuspid valve & $93(86 \%)$ & $50(98 \%)$ \\
\hline Bicuspid or unicuspid valve & $12(11 \%)$ & $1(2 \%)$ \\
\hline Rheumatic & $3(3 \%)$ & $0(0 \%)$ \\
\hline LV end-diastolic volume, mL & $80 \pm 36$ & $79 \pm 34$ \\
\hline LV end-systolic volume, mL & $33 \pm 30$ & $34 \pm 28$ \\
\hline LVEF, \% & $63 \pm 12$ & $61 \pm 12$ \\
\hline LVEF < 50\%, N (\%) & $13(12 \%)$ & $9(18 \%)$ \\
\hline LV mass index, $\mathrm{g} / \mathrm{m}^{2}$ & $113 \pm 38$ & $124 \pm 36$ \\
\hline Relative wall thickness & $0.42 \pm 0.07$ & $0.43 \pm 0.07$ \\
\hline USH, N (\%) & $50(46 \%)$ & $24(22 \%)$ \\
\hline Left atrial volume index, $\mathrm{mL} / \mathrm{m}^{2}$ & $46 \pm 14$ & $47 \pm 12$ \\
\hline $\mathrm{E}, \mathrm{cm} / \mathrm{s}$ & $75.7 \pm 26.0$ & $75.1 \pm 24.2$ \\
\hline $\mathrm{E} / \mathrm{A}$ & $0.80 \pm 0.33$ & $0.76 \pm 0.32$ \\
\hline$e^{\prime}, \mathrm{cm} / \mathrm{s}$ & $5.2 \pm 2.2$ & $4.7 \pm 1.5$ \\
\hline$E / e^{\prime}$ & $15.2 \pm 7.4$ & $16.4 \pm 6.9$ \\
\hline
\end{tabular}


TEE, transesophageal echocardiography; LV, left ventricular; EF, ejection fraction; USH, upper septal hypertrophy; E, LV early-diastolic inflow velocity; E/A, ratio of $E$ to LA systolic velocity; e', early-diastolic mitral annular velocity; E/e', the ratio of $E$ to e'.

\section{Table 2. Echocardiographic findings}

\begin{tabular}{|lll|}
\hline & All $(\mathrm{N}=108)$ & TEE cohort $(\mathrm{N}=51)$ \\
\hline LVOT TVI, $\mathrm{mm}$ & $21 \pm 2$ & $23 \pm 7$ \\
\hline LVOT velocity, $\mathrm{m} / \mathrm{s}$ & $1.03 \pm 0.33$ & $0.99 \pm 0.33$ \\
\hline $\mathrm{SV}_{\mathrm{Cont}}, \mathrm{mL}$ & $77.2 \pm 21.8$ & $73.6 \pm 23.1$ \\
\hline $\mathrm{SV}_{\mathrm{PISA}}, \mathrm{mL}$ & $66.3 \pm 17.9$ & $63.2 \pm 15.5$ \\
\hline EOA $_{\text {Cont, }} \mathrm{cm}^{2}$ & $0.86 \pm 0.33$ & $0.72 \pm 0.29$ \\
\hline EOA $_{\mathrm{PISA}}, \mathrm{cm}^{2}$ & $0.75 \pm 0.29$ & $0.62 \pm 0.22$ \\
\hline GOA, $\mathrm{cm}^{2}$ & & $0.69 \pm 0.16$ \\
\hline Peak aortic valve velocity, $\mathrm{m} / \mathrm{s}$ & $4.2 \pm 1.0$ & $4.7 \pm 0.8$ \\
\hline Mean aortic valve gradient, $\mathrm{mmHg}$ & $43.9 \pm 21.4$ & $53.3 \pm 20.0$ \\
\hline
\end{tabular}

LV, left ventricular; LVOT, LV outflow tract; TVI, time velocity index; $\mathrm{SV}_{\text {Cont }}$, stroke volume measured using the Doppler method; $S V_{P I S A}$, stroke volume measured using the proximal isovelocity surface area method; $E_{\text {COnt }}$, effective orifice area calculated using the continuity equation; EOA $A_{P I S A}$, effective orifice area calculated using the PISA method; GOA, geometric orifice area.

\section{Table 3 Comparisons of stroke volume according to LV morphology and LVOT flow velocity}




\begin{tabular}{|cllllll|}
\hline \multicolumn{3}{|l}{ Upper septal hypertrophy } & & \multicolumn{3}{l|}{ LVOT flow velocity } \\
& Presence & Absence & P value & $>0.96 \mathrm{~m} / \mathrm{s}$ & $£ 0.96 \mathrm{~m} / \mathrm{s}$ & P value \\
& $(\mathrm{N}=50)$ & $(\mathrm{N}=58)$ & & $(\mathrm{N}=53)$ & $(\mathrm{N}=55)$ & \\
$\mathrm{SV}_{\text {Cont }} \mathrm{mL}$ & $87.4 \pm 21.3$ & $68.5 \pm 18.2$ & $<0.001$ & $87.7 \pm 21.4$ & $67.1 \pm 16.9$ & $<0.001$ \\
$\mathrm{SV}_{\mathrm{PISA}}, \mathrm{mL}$ & $68.2 \pm 22.1$ & $64.6 \pm 13.3$ & 0.329 & $69.8 \pm 20.6$ & $62.9 \pm 14.1$ & 0.045 \\
\hline
\end{tabular}

Abbreviations are the same as Table 2.

\section{Figures}
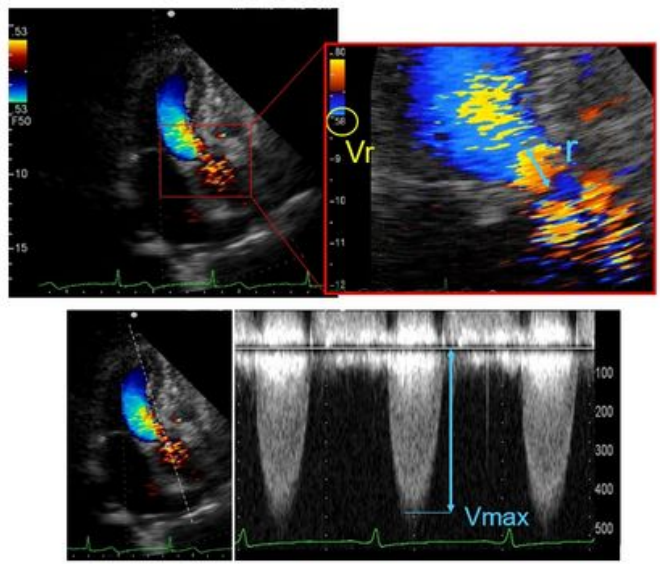

$\mathrm{EOA}_{\mathrm{PISA}}=2 \pi r^{2} \times \mathrm{Vr} / \mathrm{Vmax}$

A
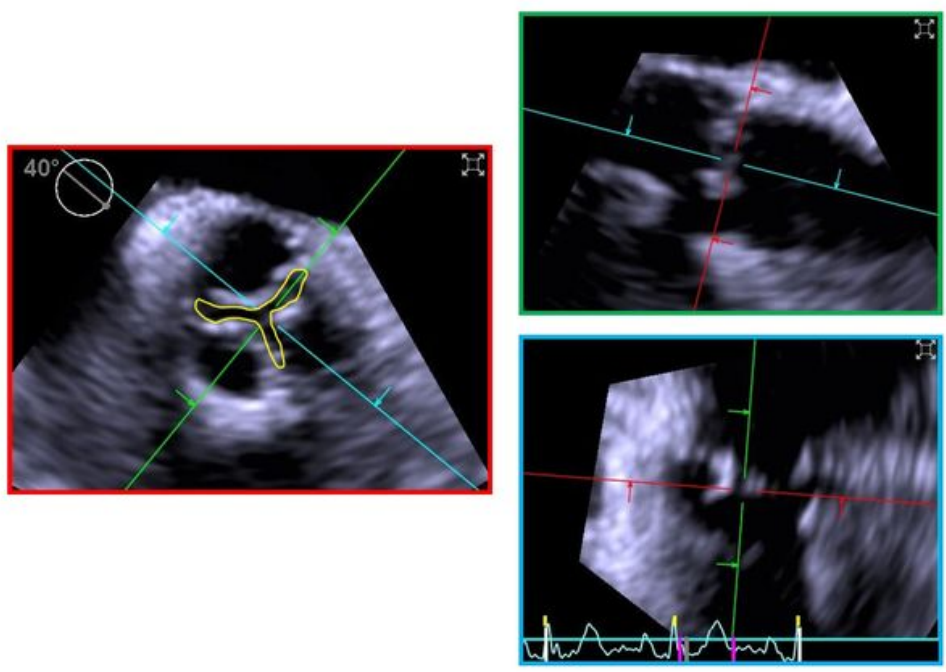

B

\section{Figure 1}

Measurement of the PISA radius (A, upper panel) and trans-aortic valvular peak velocity (A, lower panel) for estimation of EOA and analysis of three-dimensional transesophageal echocardiographic data (B) A: Proximal isovelocity surface was visualized using a zoom mode and radius of PISA $(r)$ was measured. Transvalvular flow rate was then calculated as $2 \Pi r 2$ * aliasing velocity $(\mathrm{Vr})$ and EOAPISA was defined as flow rate/peak transvalvular velocity (Vmax). B: From the three-dimensional volume data set, the shortaxis view of the aortic valve orifice (left panel) was adjusted by using the corresponding long-axis plane (right upper panel) and coronal plane (right lower panel). The geometrical orifice area was then measured 
at the mid-systolic frame by tracing the inside of the calcified cusps. PISA, proximal isovelocity surface area; EOA, effective orifice area; EOAPISA, EOA calculated using the PISA method.
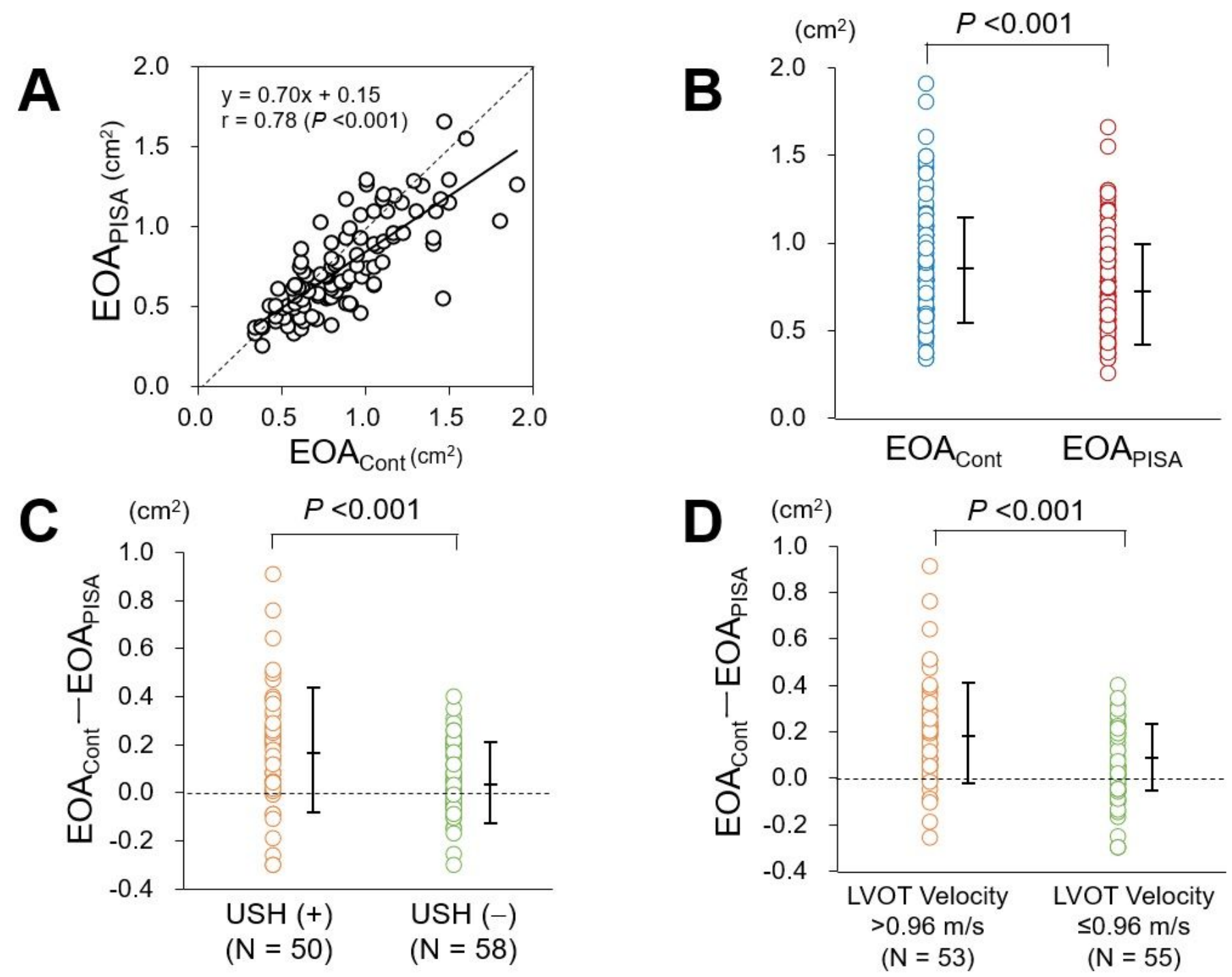

\section{Figure 2}

Comparisons of EOACont and EOAPISA A: Correlation between EOACont and EOAPISA showing an acceptable agreement between the parameters. B: Comparison of between EOACont and EOAPISA showing a slightly but significantly smaller EOAPISA than EOACont. C and D: Comparison of difference between EOACont and EOAPISA between patients with and without USH (C) and those with and without high LVOT flow velocity (D). EOACont, EOA calculated using continuous equation; USH, upper septal hypertrophy; LVOT, left ventricular outflow tract. Other abbreviations are the same as Figure 1. 

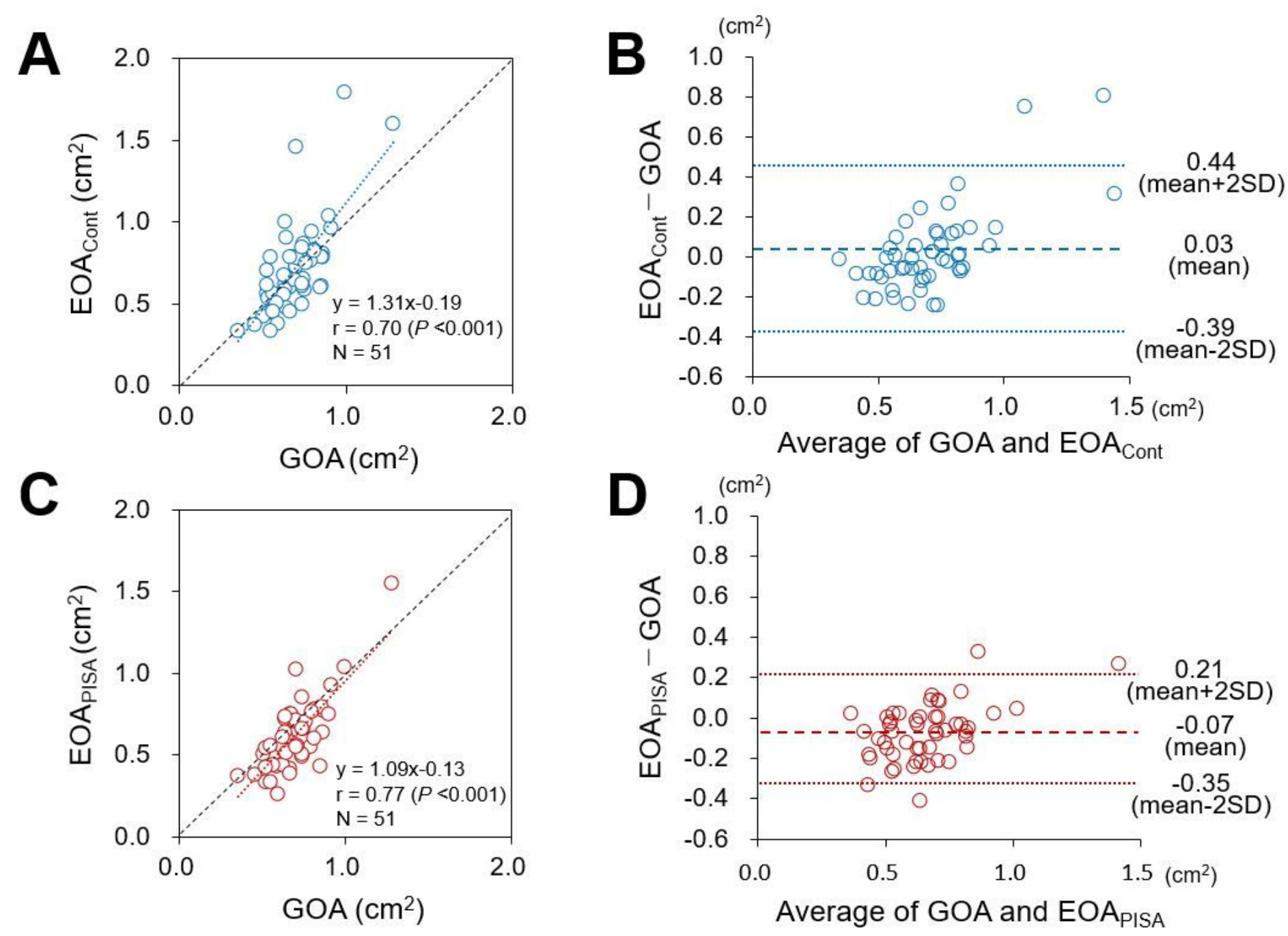

Figure 3

Relationships of EOA to GOA Scatter plots and Bland-Altman plots of relationship between EOACont and GOA ( $A$ and $B$ ) and that between EOAPISA and GOA ( $C$ and D) are shown. Note that negative bias, which should exist between EOA and GOA was observed only in between EOAPISA and GOA. GOA, geometrical orifice area. Other abbreviations are the same as Figure 1 and 2. 


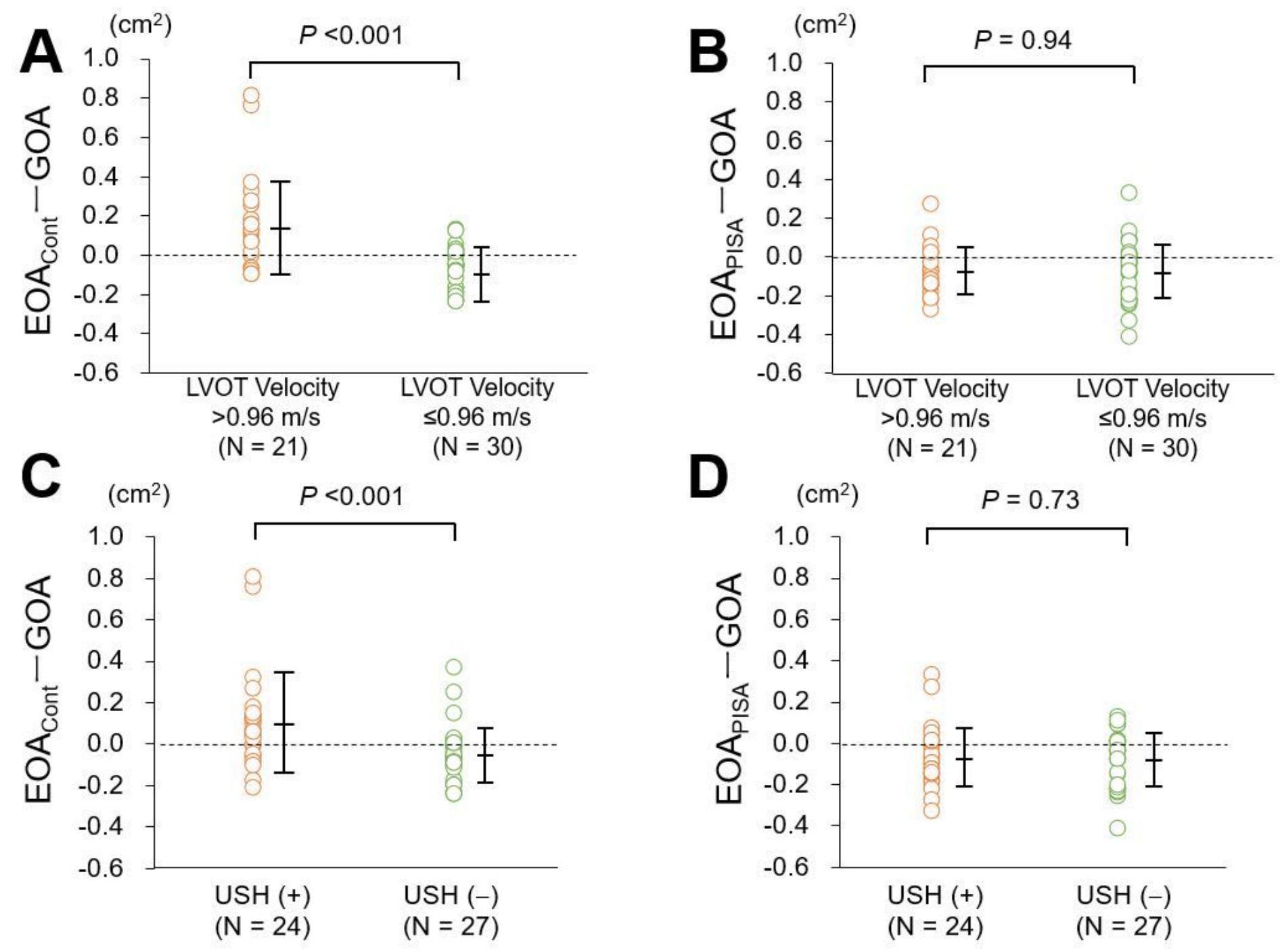

Figure 4

Comparison of differences in EOA and GOA between patients with and without high LVOT flow velocity (A and $\mathrm{B}$ ) and between patients with and without USH (C and D). Abbreviations are the same as Figures 1, 2, and 3 . 

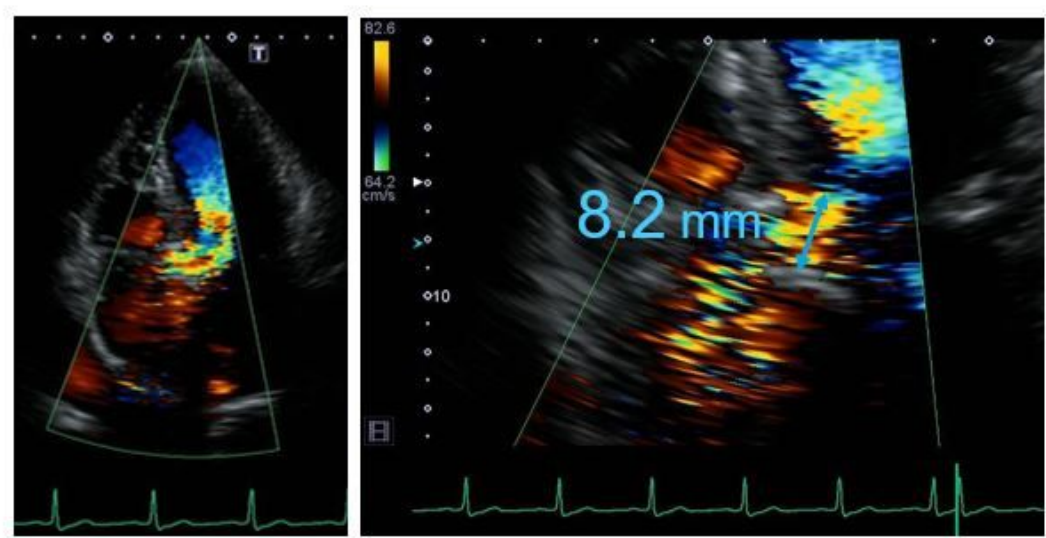

$E_{\text {PISA }}: 0.55 \mathrm{~cm}^{2}$
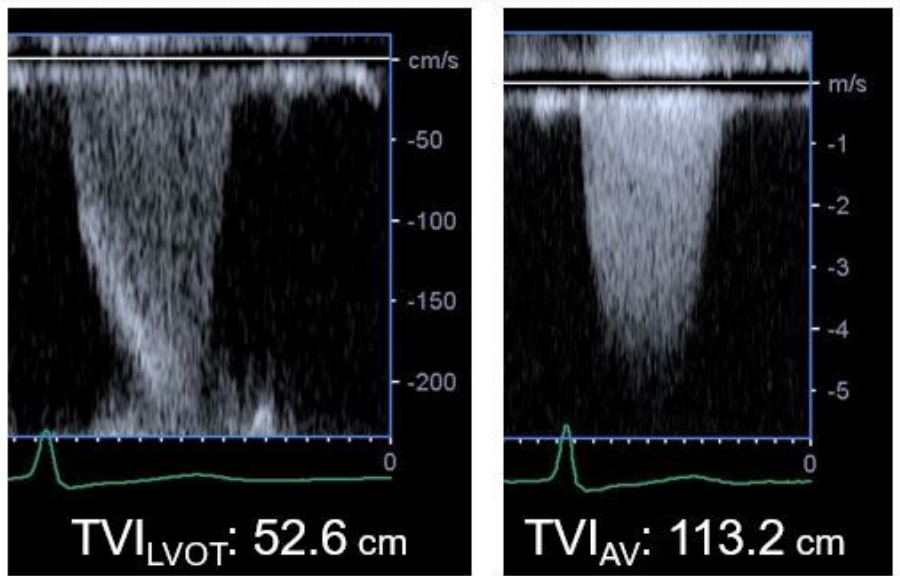

$E_{\text {Cont }}: 1.46 \mathrm{~cm}^{2}$

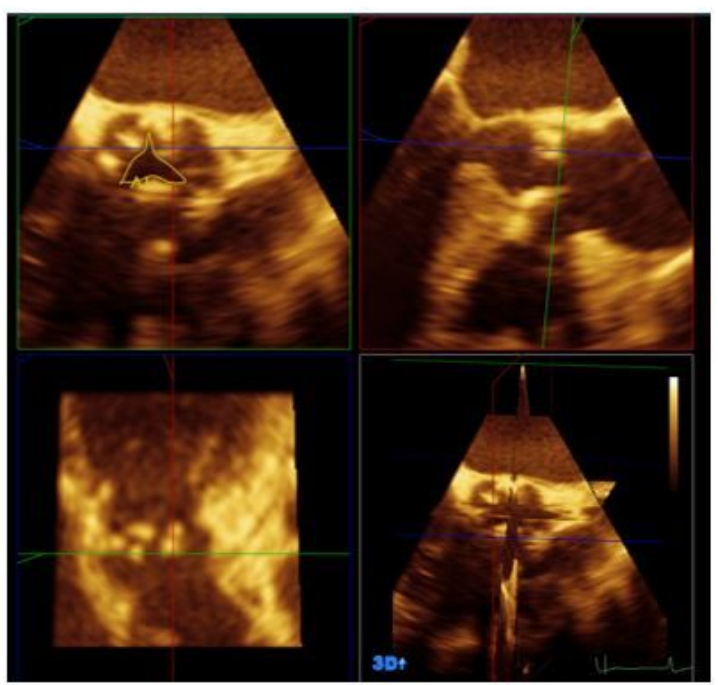

GOA: $0.70 \mathrm{~cm}^{2}$

\section{Figure 5}

A case showing considerable discrepancy between EOACont and GOA Owing to the high LVOT flow velocity, stroke volume was estimated as $164 \mathrm{~mL}$ in the calculation process of continuity equation and EOACont was finally calculated as $1.46 \mathrm{~cm} 2$, which was two-times larger than the GOA. In contrast, EOAPISA was calculated as $0.55 \mathrm{~cm} 2$ from the PISA radius of $8.2 \mathrm{~mm}$, aliasing velocity of $64.2 \mathrm{~cm} / \mathrm{s}$, and peak transvalvular velocity of $488 \mathrm{~cm} / \mathrm{s}$. TVILVOT, time velocity integral of the LVOT; TVIAV, time velocity integral of the maximal aortic valve flow. Other abbreviations are the same as Figure 1, 2, and 3. 